\title{
INVARIANCE THEORY OF HERMITIAN MANIFOLDS
}

\author{
HAROLD DONNELLY
}

\begin{abstract}
We prove that the invariants of a Hermitian manifold are generated by contractions in the components of the curvature and torsion tensors of the canonical Hermitian connection.
\end{abstract}

Introduction. Let $P$ be a map from Hermitian manifolds $M$ to complex valued functions on $M$. We say that $P$ is an invariant of order $k$ in the derivatives of the metric if for any $m \in M$ and holomorphic coordinate system normalized at origin $m, h_{\alpha \bar{\beta}}(m)=\delta_{\alpha \beta}, P$ may be expressed as a polynomial in the derivatives of $h_{\alpha \bar{\beta}}$ with respect to $\partial / \partial z_{\gamma}, \partial / \partial \bar{z}_{\gamma}$ such that any monomial of $P$ contains precisely $k$ derivatives. These invariants are important in applications to the spectral geometry of complex manifolds as is clearly explained in [4].

Our main result is that the invariants described above are given by contractions in the components of the curvature and torsion tensors of the canonical Hermitian connection and their covariant derivatives with respect to this connection. We then calculate the invariants of order two on any Hermitian manifold and the invariants of order two and four on a Kaehler manifold. The result agrees with computations by Gilkey [4], [5] who used different methods.

Our approach seems more consistent with classical differential geometry than that of Gilkey. We also believe it should facilitate the computation of higher order invariants.

The convention of summing over repeated indices is adopted throughout.

1. Geodesic coordinates. Suppose $(M, g)$ is a Riemannian manifold with a connection $\nabla$ on its tangent bundle preserving $g$. Then relative to a geodesic coordinate system the components $g_{i j}$ of the metric tensor have a formal Taylor series whose coefficients may be expressed in terms of the components of the curvature and torsion tensors of $\nabla$ and their covariant derivatives. This fact is essentially well known. For completeness we present a proof following [1] and introducing the necessary modifications since our connection need not have torsion zero.

Received by the editors October 30, 1974 and, in revised form, December 15, 1974 and February 10, 1975.

AMS (MOS) subject classifications (1970). Primary 53C99. 
Let $x$ be a geodesic coordinate system and let $s_{i}$ be the orthonormal frame obtained from $\partial / \partial x^{i}$ at $p$ by parallel transport along radial geodesics through $p$. The dual frame to $s_{i}$ is therefore a frame of 1-forms $\theta^{i}$ well defined near $p$. The connection forms relative to $s_{i}$ will be denoted by $\theta_{j}^{i}$ and the radial field by $R$.

Recall the structure equations

$$
\begin{aligned}
& d \theta^{i}=\theta_{j}^{i} \wedge \theta^{j}+S_{j k}^{i} d x^{j} \wedge d x^{k}, \\
& d \theta_{j}^{i}=\theta_{k}^{i} \wedge \theta_{j}^{k}+K_{j k l}^{i} d x^{k} \wedge d x^{l}
\end{aligned}
$$

where $S_{j k}^{i}$ and $K_{j k l}^{i}$ denote the components of the torsion and curvature tensors.

We have the formulas

$$
i(\Re) \theta^{i}=x^{i}, \quad i(\Re) \theta_{j}^{i}=0, \quad g_{i j} d x^{i} \otimes d x^{j}=\theta^{\alpha} \otimes \theta^{\alpha} .
$$

Introduce functions $a_{j}^{i}$ relating $\theta^{i}$ and $d x^{i}$ :

$$
\theta^{i}=a_{j}^{i} d x^{j} .
$$

Then it is sufficient to determine the $a$ 's in terms of torsion and curvature since $g_{i j}=a_{i}^{\alpha} a_{j}^{\alpha}$. Applying $\Re$ to $\theta^{i}$ yields

$$
\begin{aligned}
\Re \theta^{i} & =i(\Re) d \theta^{i}+d x^{i} \\
& =i(\Re)\left[\theta_{j}^{i} \wedge \theta^{j}\right]+i(\Re)\left[S_{j k}^{i} d x^{j} \wedge d x^{k}\right]+d x^{i}
\end{aligned}
$$

using the first structure equation. Then

$$
\Re \theta^{i}=-\theta_{j}^{i} x^{j}+2 S_{j k}^{i} x^{j} d x^{k}+d x^{i} .
$$

Next apply $(r)(\Re)(1 / r)$ obtaining

$$
\begin{aligned}
(r)(\Re)\left(\frac{1}{2}\right)\left(\Re \theta^{i}\right) & =-i(\Re) d \theta_{j}^{i} x^{j}+2 \Re\left(S_{j k}^{i}\right) x^{j} d x^{k}+2 S_{j k}^{i} x^{j} d x^{k} \\
& =-2 K_{j k l}^{i} x^{j} x^{k} d x^{l}+2 \Re\left(S_{j k}^{i}\right) x^{j} d x^{k}+2 S_{j k}^{i} x^{j} d x^{k}
\end{aligned}
$$

using the second structure equation.

On the other hand, computing in terms of the $a$ 's:

$$
\Re \theta^{i}=\left(\Re a_{j}^{i}+a_{j}^{i}\right) d x^{j}, \quad(r)(\Re)(1 / r) \theta^{i}=\left(\Re^{2} a_{j}^{i}+\Re a_{j}^{i}\right) d x^{j} .
$$

Hence equating coefficients one obtains the relation

$$
\left(\Re^{2}+\Re\right) a_{l}^{i}=-2 K_{j k l}^{i} x^{j} x^{k}+(2 \Re+2) S_{j l}^{i} x^{j} .
$$

Let us now write $\hat{a}, \hat{K}$, etc. for the formal Taylor series relative to $x$ about $p$ of the function indicated and $\hat{a}[n], \hat{K}[n]$, etc. for the term of homogeneity $n$ in 
this expansion. Then by Euler's formula $\mathcal{R}$ preserves homogeneity and multiplies $\hat{a}[n]$ by $n$.

Thus (1.1) yields the explicit formula:

$$
\left(n^{2}+n\right) \hat{a}_{l}^{i}[n]=-2 x^{j} x^{k} \hat{K}_{j k l}^{i}[n-2]+(2 n+2) \hat{S}_{j l}^{i}[n-1] x^{j} .
$$

2. Hermitian connection on complex manifolds. In this section we recall some well-known facts concerning complex manifolds following the account in [6].

Suppose $H$ is a Hermitian metric on the tangent bundle $T(X)$ of $X$ a complex manifold. If $g$ is the underlying real metric, then by definition

$$
g(X, Y)=\text { real part of } H(X, Y) ;
$$

we extend $g$ to the complexified tangent bundle to be conjugate linear. Define $g_{\alpha \bar{\beta}}=g\left(\partial / \partial z_{\alpha}, \partial / \partial z_{\beta}\right)$ and $g^{\alpha \bar{\beta}}$ the inverse matrix of $g_{\alpha \bar{\beta}}$.

Consider the associated Hermitian connection on the holomorphic tangent bundle. This is defined by the requirements that the metric tensor is parallel and the connection form of type $(1,0)$. Extend the covariant derivative to be complex linear on the complexified tangent bundle.

Then we have the following formulas for covariant differentiation:

$$
\nabla_{\partial / \partial z_{\alpha}}\left(\partial / \partial z_{\beta}\right)=\sum \Gamma_{\alpha \beta}^{\gamma} \partial / \partial z_{\gamma}, \nabla_{\partial / \partial \bar{z}_{\alpha}}\left(\partial / \partial z_{\beta}\right)=0,
$$

where $\Gamma_{\alpha \beta}^{\gamma}=\sum\left(\partial g_{\beta \bar{\varepsilon}} / \partial z_{\alpha}\right) g^{\varepsilon \bar{\gamma}}$.

The torsion tensor is given by

$$
S_{\alpha \beta}^{\gamma}=\Gamma_{\alpha \beta}^{\gamma}-\Gamma_{\beta \alpha}^{\gamma} .
$$

$S$ vanishes if and only if the manifold is Kaehler.

The nonvanishing components of the curvature tensor are

$$
\begin{array}{ll}
K_{\gamma \alpha \bar{\beta}}^{\delta}=\partial \Gamma_{\alpha \gamma}^{\delta} / \partial \bar{z}_{\beta}, & K_{\bar{\gamma} \alpha \bar{\beta}}^{\delta}=\partial \bar{\Gamma}_{\beta \gamma}^{\delta} / \partial z_{\alpha}, \\
K_{\gamma \bar{\beta} \alpha}^{\delta}=-K_{\gamma \alpha \bar{\beta}}^{\delta}, & K_{\bar{\gamma} \bar{\beta} \alpha}^{\delta}=-K_{\bar{\gamma} \alpha \bar{\beta}}^{\delta}
\end{array}
$$

where $\bar{\Gamma}_{\beta \gamma}^{\delta}$ denotes the complex conjugate of $\Gamma_{\beta \gamma}^{\delta}$.

We recall some identities which will be required in the sequel. These may be verified by explicit expansion in terms of the derivatives of the metric.

$$
\begin{gathered}
S_{\alpha \beta}^{\gamma}=-S_{\beta \alpha}^{\gamma} . \\
\sum\left(K_{\delta \alpha \bar{\alpha}}^{\delta}-K_{\alpha \delta \bar{\alpha}}^{\delta}\right)=\sum S_{\delta \alpha, \bar{\alpha}}^{\delta}+\frac{1}{2} \sum S_{\delta \alpha}^{\varepsilon} \bar{S}_{\delta \alpha}^{\varepsilon} .
\end{gathered}
$$

Finally if $X, Y$ are vector fields the endomorphism $K(Y, X)$ of $T M$ extends naturally to tensors of arbitrary type and

$$
\left(\nabla_{X} \nabla_{Y}-\nabla_{Y} \nabla_{X}-\nabla_{[X, Y]}\right) T=K(Y, X) T
$$


where $T$ is any tensor. $K(Y, X) T$ may be written explicitly in terms of contraction of the components of $K$ and $T$.

If our Hermitian manifold is Kaehler we have the additional identities:

$$
\begin{aligned}
K_{\beta \gamma \bar{\varepsilon}}^{\alpha}-K_{\gamma \beta \bar{\varepsilon}}^{\alpha} & =0 . \\
K_{\beta \gamma \overline{\bar{\varepsilon}} \bar{\delta}}^{\alpha}-K_{\beta \gamma \bar{\delta}, \bar{\varepsilon}}^{\alpha} & =0 . \\
K_{\gamma \alpha \bar{\beta}}^{\delta}-K_{\gamma \alpha \bar{\delta}}^{\beta} & =0 .
\end{aligned}
$$

3. Invariance theory for Hermitian manifolds. Since $h(X, Y)=g(X, Y)$ $+\sqrt{-1} g(X, J Y)$ where $h$ is any Hermitian metric, $g$ the underlying Riemannian metric, and $J$ the almost complex structure, invariant polynomials in the derivatives $\partial / \partial z_{\gamma}, \partial / \partial \bar{z}_{\gamma}$ of the Hermitian metric are the same as invariant polynomials in the derivatives $\partial / \partial z_{\gamma}, \partial / \partial \bar{z}_{\gamma}$ of the underlying Riemannian metric.

The Hermitian connection (2) induces a connection on the real tangent bundle since it preserves the complex structure. Also the exponential map of this connection provides a holomorphic coordinate chart again because the connection preserves the complex structure. By (1) the components of $g$ in this coordinate system have Taylor series whose coefficients are the components of the curvature and torsion tensor and their covariant derivatives. Thus any invariant polynomial in the derivatives of the metric is a polynomial in the components of these tensors.

At this point we recall the fundamental theorem of $U(n)$ invariance theory.

THEOREM. Let $W$ be the standard representation of $U(m)$ on $C^{m}$ and denote by $W^{*}$ its dual module $\operatorname{Hom}_{C}(W, C)$. Then the $C$ module

$$
\operatorname{Hom}_{U(m)}\left[\otimes^{r} W \otimes^{s} W^{*} ; C\right]
$$

vanishes for $r \neq s$ and for $r=s$ is spanned by elementary contractions of the type

$$
\phi_{\sigma}: W_{1} \otimes \cdots \otimes W_{r} \otimes W_{1}^{*} \otimes \cdots \otimes W_{r}^{*} \rightarrow\left\langle W_{1}, W_{\sigma(1)}^{*}\right\rangle \cdots\left\langle W_{r}, W_{\sigma(r)}^{*}\right\rangle
$$

for $r=s$ with $\sigma$ a permutation of $(1, \ldots, r)$.

This gives our main result:

THEOREM. The invariant polynomials in the derivatives of the metric on a Hermitian manifold are given by contractions in the components of the curvature and torsion tensors of the canonical Hermitian connection and their covariant derivatives with respect to this connection.

4. Invariants of order two for Hermitian manifolds. By explicitly writing out all contractions of the required order and reducing modulo the identities (2.1), (2.2), (2.3) we deduce the following basis for the invariants of order two: 


$$
\begin{array}{ll}
A=K_{\alpha \beta \bar{\beta}}^{\alpha}, & C=S_{\alpha \beta}^{\gamma} \bar{S}_{\alpha \beta}^{\gamma}, \\
B=S_{\gamma \alpha, \bar{\alpha}}^{\gamma}, & D=S_{\gamma \beta}^{\gamma} \bar{S}_{\alpha \beta}^{\alpha} .
\end{array}
$$

These invariants are a basis on a generic complex manifold. In fact they form a basis if the complex dimension of $M$ is greater than two. On complex two manifolds there are three independent invariants $A, B, C$ and $D=\frac{1}{2} C$. If the complex dimension is one than $A$ is a basis and $B=C=D=0$.

Our invariants are related to those of Gilkey [4] by $A=K+K_{1}, B$ $=-K_{1}+2 K_{2}, C=2 K_{2}, D=K_{2}+2 K_{3}$.

5. Invariants of orders two and four on a complex Kaehler manifold. $A$ gives a basis for the invariants of order two on a complex Kaehler manifold.

The invariants

$$
\begin{array}{ll}
E=\left(K_{\alpha \beta \bar{\beta}}^{\alpha}\right)^{2}=A^{2}, & G=K_{\delta \alpha \bar{\beta}}^{\delta} \bar{K}_{\gamma \alpha \bar{\beta}}^{\gamma}, \\
F=K_{\gamma \alpha \bar{\beta}}^{\delta} \bar{K}_{\gamma \alpha \bar{\beta}}^{\delta}, & H=K_{\alpha \beta \bar{\beta}, \gamma \bar{\gamma}}^{\alpha},
\end{array}
$$

form a basis on a Kaehler manifold of complex dimension greater than one. This follows by writing out all contractions and reducing by the identities $S=0$ and (2.1)-(2.7). On a Riemann surface $E, H$ form a basis and $E=F=G$.

Our invariants are related to the invariants of Gilkey and Sacks [5] by $\tau^{2}=E, \Delta \tau=2 H$,

$$
\begin{aligned}
& *\left(\Omega^{m-2} \wedge C_{2}\right)=1 / 32 \pi^{2}(F-4 G+E), \\
& *\left(\Omega^{m-2} \wedge C_{1}^{2}\right)=1 / 16 \pi^{2}(E-2 G) .
\end{aligned}
$$

\section{BIBLIOGRAPHY}

1. M. Atiyah, R. Bott and V. K. Patodi, On the heat equation and index theorem, Invent. Math. 19 (1973), 279-330.

2. H. Donnelly, Minakshisundaram's coefficients on Kaehler manifolds, Proc. Sympos. Pure Math., vol. 27, part 2, Amer. Math. Soc., Providence, R.I., 1975, pp. 195-203.

3. - A spectral condition determining the Kaehler property, Proc. Amer. Math. Soc. 47 (1975), 187-194.

4. P. Gilkey, Spectral geometry and the Kaehler condition for complex manifolds, Invent. Math. 26 (1974), 231-258.

5. P. Gilkey and J. Sacks, Spectral geometry and manifolds of constant holomorphic sectional curvature, Proc. Sympos. Pure Math., vol. 27, part 2, Amer. Math. Soc., Providence, R.I., 1975, pp. 281-285.

6. V. K. Patodi, An analytic proof of the Riemann-Roch-Hirzebruch theorem for Kaehler manifolds, J. Differential Geometry 5 (1971), 251-283. MR 44 \# 7502.

Department of Mathematics, Massachusetts Institute of Technology, Cambridge, MASSACHUSETTS 02139 